\title{
Fatal Urosepsis: A 41 Year-Old Pregnant Woman-Case Report
}

\author{
Malgorzata Gosciniak ${ }^{1}$ Dariusz Kawecki2 ${ }^{*}$, Malgorzata Miklaszewska ${ }^{3}$, \\ Zenon Truszewski ${ }^{4}$, Tomasz Lazowski ${ }^{3}$, Miroslaw Wielgos ${ }^{4}$, Piotr Radziszewski ${ }^{5}$ \\ ${ }^{1}$ Department of Emergency Medicine, Medical University of Warsaw, Warsaw, Poland \\ ${ }^{2}$ Department of Medical Microbiology, Warsaw Medical University, Warsaw, Poland \\ ${ }^{3}$ Department of Intensive Care, Medical University of Warsaw, Warsaw, Poland \\ ${ }^{4}$ Department of Obstetrics and Gynecology, Medical University of Warsaw, Warsaw, Poland \\ ${ }^{5}$ Department of General, Oncologic and Functional Urology, Medical University of Warsaw, Warsaw, Poland \\ Email: ${ }^{*}$ dkawecki@o2.pl
}

Received 9 October 2014; revised 10 November 2014; accepted 18 November 2014

Copyright (C) 2014 by authors and Scientific Research Publishing Inc.

This work is licensed under the Creative Commons Attribution International License (CC BY).

http://creativecommons.org/licenses/by/4.0/

(c) (i) Open Access

\begin{abstract}
Introduction: Urinary tract infections are one of the most frequent infectious complications during pregnancy [1], while acute pyelonephritis is the main non-obstetric reason for hospitalisation [2] [3] and septic shock in pregnant women. Objectives and methods: The aim of our study is to analyse the diagnostics and treatment of a 41-year-old pregnant woman with the most severe form of urinary tract infection-urosepsis. Case report: A 41-year old woman in the 12th week of pregnancy hospitalised at Intensive Care Unit (ICU) after urological intervention (JJ stent). On the first day after the intervention, the patient was diagnosed as having urosepsis. Scores: SOFA 14 pts, APACHE II 26 pts, SAPS II SCORE 61 pts. Second day in ICU: spontaneous abortion. Microbiological investigation: Escherichia coli-extended-spectrum beta-lactamase (ESBL+). Conclusions: A pregnant patient with a complicated infection of the urinary system ought to be treated in a multi-profile hospital, offering a possibility to consult doctors of various specialisations (urologist, gynaecologist, anaesthesiologist, nephrologist) as well as full access to a radiology laboratory, which will ensure the choice of appropriate and safe treatment for both the mother and the developing foetus.
\end{abstract}

\section{Keywords}

Pregnancy, Urosepsis, Nephrolithiasis, Ureteroscopy

\footnotetext{
${ }^{*}$ Corresponding author.
}

How to cite this paper: Gosciniak, M., Kawecki, D., Miklaszewska, M., Truszewski, Z., Lazowski, T., Wielgos, M. and Radziszewski, P. (2014) Fatal Urosepsis: A 41 Year-Old Pregnant Woman-Case Report. Open Journal of Urology, 4, 137-141. http://dx.doi.org/10.4236/oju.2014.411024 


\section{Introduction}

The anatomical and physiological changes which occur during pregnancy to ensure the optimal development of the foetus, predispose women to urinary tract infections (UTIs) [2] [4] [5] and nephrolithiasis. The dilatation of the urinary system, which progresses from the early stage of pregnancy (10th week), is attributed mainly to the mechanical compression on the ureters caused by the enlarging uterus, and in a lesser degree to the impact of hormones, most importantly progesterone [4]-[6]. Urinary tract infections are one of the most frequent infectious complications during pregnancy [1], while acute pyelonephritis is the main non-obstetric reason for hospitalisation [2] [3] [7] and septic shock in pregnant women [1].

The incidence of urinary tract infections in pregnant women is estimated at $0.3 \%$ - $1.3 \%$ [4] [8] [9], with the rate in the population of non-pregnant women of childbearing age being similar. The major difference concerns the incidence of cases in which asymptomatic bacteriuria and lower urinary tract infections progress to acute pyelonephritis [4] [6] [9]. About 15\% - 50\% of cases of cystitis [9] and 20\% - 40\% cases [8] of asymptomatic bacteriuria are complicated by upper urinary tract infections, observed in circa $2 \%$ of the pregnant women [2] [8] [9]. The classification of urinary tract infections takes into account risk factors such as: coexistent diabetes, anaemia, neurogenic bladder, vesicoureteral reflux, recurrent infections of the urinary tract before pregnancy, nephrolithiasis, previous urological interventions [2] [9]. When these occur, a UTI has to be considered as an infection with a high risk of complications, otherwise it is considered as an uncomplicated infection.

Etiological factors of urinary system infections in pregnant women do not differ from those for the population of non-pregnant women of childbearing age: in $80 \%$ - 90\% of cases, it is Escherichia coli, less frequent Klebsiella pneumoniae, Proteus mirabilis, Enterobacter species, Staphylococcus saprophyticus, Group B Streptococcus [6] [8] [9].

The most severe form of infection is urosepsis, which can be defined as systematic inflammatory response syndrome (SIRS) in reaction to a progressing infection in the urinary tract [8] [10]-[12]. Severe sepsis is diagnosed when sepsis is associated with organ dysfunction [8] [10]-[12], while in septic shock the above symptoms are so advanced that catecholamine treatment is necessary to maintain normal blood pressure [8] [10]-[12]. Refractory septic shock is diagnosed when the state of the patient does not improve in spite of more than $1 \mathrm{~h}$ of fluid therapy and pharmacological intervention [8] [10]-[12]. Urosepsis comprises circa 25\% of all sepsis cases [10]. It is usually less severe than sepsis originating from other organs. In circa 5\% of cases [10] it progresses to severe sepsis with a mortality rate of $20 \%$ - 42\% [8] [10]. The urological pathologies that are most commonly associated with cases of urosepsis in the literature are obstructive diseases of the urinary tract complicated by infection [8] [12], the cause of which is primarily attributed to nephrolithiasis [8] [12], before tumours originating in the urinary tract [12] and pregnancy [12]. Approximately 17\% of all urosepsis cases develop after urological interventions [12]. The incidence of urosepsis in pregnant women is circa 5\% [12]. The most common etiological factors for urosepsis are Gram-negative bacteria Enterobacteriaceae, although Pseudomonas spp., Grampositive bacteria and less frequent fungi may also play a role [8].

Treating urosepsis involves initially empirical antibiotic therapy, usually with broad-spectrum antibiotics, covering the most common etiological factors, then, after obtaining urine culture results, modified into directed treatment. Supportive treatment involves: fluid therapy, oxygen therapy, catecholamines, steroids, dialysis therapy [8] [10] [11] [13] [14]. An important element of the treatment is to monitor the locus of infection, which often requires a surgical intervention. This may involve, among others, relief of retention in the pyelocalyceal system in the presence of obstruction in the urinary tract and abscess drainage [8] [10] [11] [13] [14].

Diagnosing sepsis in pregnant patients may pose difficulties due to anatomic and physiological changes which may mask some symptoms and make it more difficult to interpret the results of diagnostic tests. Moreover, the response of the mother's body to the infection is changed and at the same time weakened by changes in the immunological system during pregnancy, which lead to natural immunosuppression allowing the optimal development of the foetus. Clinical observation in the first days of treatment is important due to a high risk of complications and bacteraemia episodes (circa 15\% - 20\%) [1], which can lead to severe complications threatening the life of the mother and the foetus. These include: low birth weight of the infant, preterm labour, increased maternal mortality rate, anaemia, hypertension, kidney failure, respiratory distress syndrome and sepsis [1] [3]-[5] [9].

The aim of this paper is to prove that pregnant patients with complicated infections of the urinary system should undergo treatment in a multi-profile hospital, thus enabling a timely urological intervention and decreas- 
ing the possibility of severe complications threatening the life of both the mother and the child.

\section{Case Report}

We present a case of a 41-year-old woman in the 12th week of pregnancy who was hospitalised at the Urology Clinic and the Intensive Care Unit (ICU) of the Medical University of Warsaw Clinical Hospital with symptoms of left-side renal colic accompanied with symptoms of urinary tract infection. In the interview the patient reported having undergone surgical treatment at the age of 9 due to left-side vesicoureteral reflux. She was hospitalised for 3 days at the County Hospital, then she was transferred to a higher referral level institution on account of increasing inflammatory markers and persistent pains of a colicky character. Upon admission, the general condition of the patient was good; her cardiovascular and respiratory conditions were stable: blood pressure $105 / 70 \mathrm{mmHg}$, regular pulse $102 /$ minute, temperature $36.6^{\circ} \mathrm{C}$. The patient reported minor dysuria symptoms and pain of a left-side renal colic character. Laboratory tests revealed significantly increased inflammatory markers: CRP 390 mg/L, procalcitonin $81 \mathrm{ng} / \mathrm{mL}$, leucocytosis WBC > $20 \mathrm{G} / \mathrm{l}$, moreover peripheral blood count revealed microcytic anaemia with low iron levels and thrombocytopenia $90 \mathrm{~g} / \mathrm{L}$. An ultrasonography of the abdomen performed in the first day of hospitalisation showed a $10 \mathrm{~mm}$ calculus in the end part of the left ureter, accompanied by dilatation of the pyelocalyceal system on the left side and a potential double pyelocalyceal system. Due to high inflammation markers the empirical antibiotic therapy was broadened: Amikacin $(1 \mathrm{G})$ was added to peracillin $(4 \mathrm{G})+\operatorname{tazobactam}(0.5 \mathrm{G})$ four times daily, while fluid therapy, diastolic-analgetic and antifebrile treatment were continued. No response or improvement in the clinical condition of the patient was achieved. Owing to the ineffectiveness of conservative treatment, at the end of the first day of hospitalisation it was decided that surgical intervention was necessary and URS was performed under general anaesthesia. During ureteroscopy the calculus was removed from the left ureter, a 9 Fr JJ stent was inserted in the pyelocalyceal system of the left kidney and bloody purulent urine was drained.

Several hours after the surgical treatment (2nd day of hospitalisation) the general condition of the patient worsened, showing symptoms of respiratory failure with tachypnea 30/min, tachycardia 136/minute and blood pressure 115/70 mmHg; in physical examination, crepitation was heard over the whole lungs; laboratory tests revealed further increase of the inflammation markers (CRP, PCT), progressing thrombocytopenia, coagulation abnormalities and increased lactate concentration. While pulmonary circulation was stable, chest X-ray revealed mottled densities extending from the hilum, corresponding to inflammatory changes. The presence of the densities was confirmed the following day by CT chest scan. On the second day of hospitalisation, the pregnant patient was transferred to ICU. Due to increasing hypoxemia $\left(\mathrm{PO}_{2} 47 \mathrm{mmHg}\right)$ the patient required intubation and mechanical ventilation as well as infusion of catecholamines to aid the circulation system because of hypotension (RR 80/45 mmHg). On the basis of overall clinical picture and the results of additional tests urosepsis complicated by septic shock was diagnose, with severity assessed in the first day at the ICU with the following scores: 14 SOFA, 26 APACHE II, 16 SAPS II SCORE.

In the second day of the patient's stay at the ICU, spontaneous abortion took place, accompanied with heavy bleeding from the genital tract, which aggravated the previously observed anaemia, with Hgb falling to $77 \mathrm{~g}$ and Hct to $0.229 \mathrm{~L} / \mathrm{L}$, and required transfusion of 3u of packed red blood cells. Following gynaecological consultation, dilation and curettage was performed, and oxytocin and methergine were administered, which stopped the bleeding from the genital tract. Despite combined empirical antibiotic therapy, neither decrease in inflammation markers nor improvement in the general condition of the patient was achieved: she still had high fever up to $40^{\circ} \mathrm{C}$. After extended-spectrum beta-lactamase -positive (ESBL+) Escherichia coli was grown in blood and urine culture, the antibiotic therapy was modified into directed therapy, in accordance with the antibiogram results: peracillin + tazobactam and amikacin were discontinued, while cylastatyna + imipenem $0.5 \mathrm{G}$, four times daily, and Clindamycin $0.3 \mathrm{G}$ three times daily, were introduced, obtaining a decrease in laboratory markers of the infection, the fever dropping to $38^{\circ} \mathrm{C}$. Follow-up ultrasonography of the abdomen did not show a dilatation of the pyelocalyceal system of the kidneys and the position of the JJ stent was correct. On the 5th day at the ICU the patient was extubed and the infusion of catecholamines was discontinued. Within the following days of hospitalisation her cardiovascular and respiratory conditions were stable. On the 7th day she was transferred to the Urology Clinic for further treatment. Within the following days of hospitalisation further decrease in laboratory markers of the infection was observed and the general condition of the patient improved. Six weeks later, JJ stent was removed and the patient was recommended for periodic urological examination. Laboratory data are shown in Table 1. 
Table 1. Laboratory abnormalities.

\begin{tabular}{ccccccccc}
\hline \multirow{2}{*}{ Laboratory tests } & \multicolumn{9}{c}{ Day of hospitalisation } \\
\cline { 2 - 8 } & I & II -ICU & III & IV & V & VI & VII \\
\hline CRP mg/L & 390 & 513 & 537.50 & 185.70 & 93.40 & 45.4 & 27.4 & \\
PCT ng/mL (procalc.) & 81 & 59.52 & 36.29 & 21.08 & 14.99 & 7.67 & 19.51 \\
WBC G/L & 27.97 & 27.97 & 29.90 & 33.53 & 35.53 & 21.66 & 76 & 91 \\
PLT G/L & 90 & 62 & 75 & 73 & 73 & 117.0 & 110.0 \\
Hgb g & 109.0 & 109.0 & 77.0 & 117.0 & 117.0 & 117.0 & 0.80 \\
Creatinine mg/dL & 1.40 & 1.40 & 1.40 & 2.20 & 1.60 & 1.10 & \\
\hline
\end{tabular}

\section{Discussion}

Active diagnosing and instant treatment of urinary tract infections during pregnancy are important elements of care in pregnant patients, preventing severe complications threatening the lives of both the mother and the developing foetus. Particular attention and intensive care should be devoted to pregnant women with complicated infections of the urinary system where a delayed application of directed antibiotic therapy or postponed surgical intervention, if it is necessary, may result in initiating a systemic inflammatory response syndrome cascade, including septic shock.

The above described case of the pregnant woman confirms the fact that treating urinary system infections, especially the ones that are from the outset diagnosed as complicated, should be aggressive from the very beginning and conducted during the first days of hospitalisation. This increases the chances for a quick intervention in the case the initial antibiotic therapy is inefficient and additional factors, possibly requiring surgical treatment, appear. The described pregnant woman, apart from a vesicoureteral reflux treated in her childhood, had an additional acute pyelonephritis risk factor, namely nephrolithiasis. Altogether these factors contributed to the dramatic ending of the urinary system infection: septic shock and miscarriage. A pregnant patient with a complicated infection of the urinary system ought to be treated in a multi-profile hospital, offering a possibility to consult doctors of various specialisations (urologist, gynaecologist, anaesthesiologist, nephrologist) as well as full access to a radiology laboratory, which will ensure the choice of appropriate and safe treatment for both the mother and the developing foetus.

In the described case, the 41-year-old woman was treated for the first three days in a hospital which did not have a Department of Urology. For that reason, removing obstruction in the urinary tract through an appropriate surgical intervention was probably delayed, thus preventing a successful treatment of the infection.

The method of choice for the treatment of pregnant women with renal colic is conservative one [4] [9] [15]-[18] consisting of rehydration, analgesic and muscle relaxant treatment, and, in the case of urinary tract infection accompanying the renal colic, additional antibiotic therapy. This procedure allows for a spontaneous passage of calculi in 65\% - 85\% of patients [4] [9] [14]-[18].

Invasive treatment is applied in case of pharmacotherapy failure, ureter obstruction causing extrarenal renal failure, urosepsis, hydronephrosis in one kidney, renal colic inducting preterm labour [4] [16] [18]. As a rule, the treatment involves temporary urinary diversion with JJ stent or percutaneous nephrostomy, while the ultimate invasive treatment is delayed until the postnatal period. A surgical method alternative to temporary urinary diversion treatment in treating urolithiasis during pregnancy is ureteroscopy (URS) [4] [14] [17]-[19], which is performed in the described case. This method combines both a diagnostic and therapeutic value [5] [17]-[19]. Numerous studies on URS in pregnancy prove that it is a safe and effective method of treating urolithiasis in pregnant women in every trimester of pregnancy and the complications incidence is largely dependent on the operator's experience

The incidence of complications from URS carried out during pregnancy is similar to that in non-pregnant patients [9] [17] [20]. ESWL and PNL are contraindicated during pregnancy [16]-[18] [20].

On the basis of the case report it can be concluded that decisions on surgical interventions should be made without excessive delay in cases when obstructive diseases of the urinary tract are complicated by infection.

It should be mentioned that in the case of an already developed severe infection of the urinary system, the inflammatory reaction can be aggravated by surgical interventions performed in order to relieve retention in the 
urinary system in the presence of obstruction, as it creates a risk of an additional load of infectious agent into the blood [8]. Such was the case of the patient described above.

Considering the high mortality rate of circa $20 \%-40 \%$ for severe urosepsis [8], the difficulties in predicting its course and lack of unambiguous guidelines regarding the optimal time for introducing surgical intervention, it is most vital to prevent the illness from occurring by early and appropriate treatment of urinary tract infections and dysfunctions.

Pregnant women with a history of urological disease before pregnancy require particular attention and care so that early and appropriate treatment of urinary tract infections is ensured. In the presence of additional risk factors, patients should be treated in a hospital which offers a possibility of quick surgical intervention. Such procedures allow avoiding complications threatening the life and health of both the mother and the developing foetus.

\section{References}

[1] Morgan, J. and Roberts, S. (2013) Maternal Sepsis. Obstetrics and Gynecology Clinics of North America, 40, 69-87.

[2] Dawkins, J.C., Fletcher, H.M., Rattray, C.A., Reid, M. and Gordon-Strachan (2012) Acute Pyelonephritis in Pregnancy: A Retrospective Descriptive Hospital Based-Study. Obstetrics and Gynecology, 2012, 6 p.

[3] Gabbe, S.G., Niebyl, J.R. and Simpson, J.L. (2012) Renal Disease, Obstetrics: Normal and Problem Pregnancies. 6th Edition, Chapter 38, 875-886.

[4] Macejko, A.M. and Schaeffer, A.J. (2007) Asymptomatic Bacteriuria and Symptomatic Urinary Tract Infections during Pregnancy. Urologic Clinics of North America, 34, 35-42. http://dx.doi.org/10.1016/j.ucl.2006.10.010

[5] Fulop, T., Batuman, V., Ed. (2013) Acute Pyelonephritis, Medscape.

[6] Schnarr, J. and Smaill, F. (2008) Asymptomatic Bacteriuria and Symptomatic Urinary Tract Infections in Pregnancy. European Journal of Clinical Investigation, 38(S2), 50-57. http://dx.doi.org/10.1111/j.1365-2362.2008.02009.x

[7] Archabald, K.L., Friedman, A. and Raker, C.A. (2009) Impact of Trimester on Morbidity of Acute Pyelonephritis in Pregnancy. American Journal of Obstetrics \& Gynecology, 201, e1-e4.

[8] Grabe, M., Bjerklund-Johansen, T.E., Botto, H., Çek, M., Naber, K.G., Pickard, R.S., Tenke, P., Wagenlehner, F. and Wullt, B. (2013) Urological Infektions. EAU, 12-25, 34-38.

[9] Johnson, E.K. and Kim, E.D. (2012) Urinary Tract Infection in Pregnancy. Medscape.

[10] Wagenlehner, F.M.E., Weidner, W. and Naber, K.G. (2007) Optimal Management of Urosepsis from the Urological Perspective. International Journal of Antimicrobial Agents, 30, 390-397. http://dx.doi.org/10.1016/j.ijantimicag.2007.06.027

[11] Lucas, D.N., Robinson, P.N. and Nel, M.R. (2012) Sepsis in Obstetrics and the Role of the Anaesthetist. International Journal of Obstetrics Anaesthesia, 21, 56-67.

[12] Wagenlehner, F.M., Pilatz, A. and Weidner, W. (2011) Urosepsis-From the View of the Urologist. International Journal of Antimicrobial Agents, 38S, 51-57.

[13] Zapała, P., Dybowski, B. and Radziszewski, P. (2013) Posocznica moczowa, urosepsa, Przegląd Urologiczny.

[14] Dellinger, R.P., Carlet, J.M., Masur, H., Gerlach, H., Calandra, T., Cohen, J., Gea-Banacloche, J., Keh, D., Marshall, J.C., Parker, M.M., Ramsey, G., Zimmerman, J.L., Vincent, J.L. and Levy, M.M. (2004) Surviving Sepsis Campaign Management Guidelines Committee. Critical Care Medicine, 32, 858-873. http://dx.doi.org/10.1097/01.CCM.0000117317.18092.E4

[15] Richard Bell: Education-Tutorials Urological Problems Complications in Pregnancy. Bristol Urological Institute.

[16] Charalambous, S., Fotas, A. and Rizk, D.E.E. (2009) Urolithiasis in Pregnancy. International Urogynecology Journal, 20, 1133-1136. http://dx.doi.org/10.1007/s00192-009-0920-z

[17] Semins, M.J. and Matlaga, B.R. (2009) Ureteroscopy during Pregnancy. Indian Journal of Urology, 25, 291-295. http://dx.doi.org/10.4103/0970-1591.56173

[18] Biyani, C.S. and Joyce, A.D. (2002) Urolithiasis in Pregnancy. II: Management. BJU International, 89, 819-823. http://dx.doi.org/10.1046/j.1464-410X.2002.02773.x

[19] Bres-Niewada, E., Judycki, J., Borkowski, A. and Czaplicki, M. (2006) Ureteroskopowe leczenie ciężarnych kobiet chorych na kamicę moczowodową. Urologia Polska.

[20] Wayment, R.O., Schwartz, B.F., Ed. (2012) Pregnancy and Urolithiasis Clinical Presentation. Medscape. 
Scientific Research Publishing (SCIRP) is one of the largest Open Access journal publishers. It is currently publishing more than 200 open access, online, peer-reviewed journals covering a wide range of academic disciplines. SCIRP serves the worldwide academic communities and contributes to the progress and application of science with its publication.

Other selected journals from SCIRP are listed as below. Submit your manuscript to us via either submit@scirp.org or Online Submission Portal.
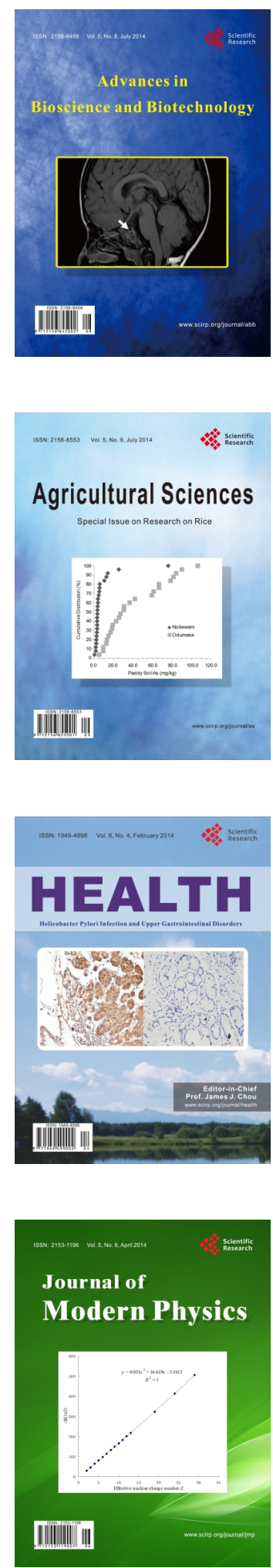
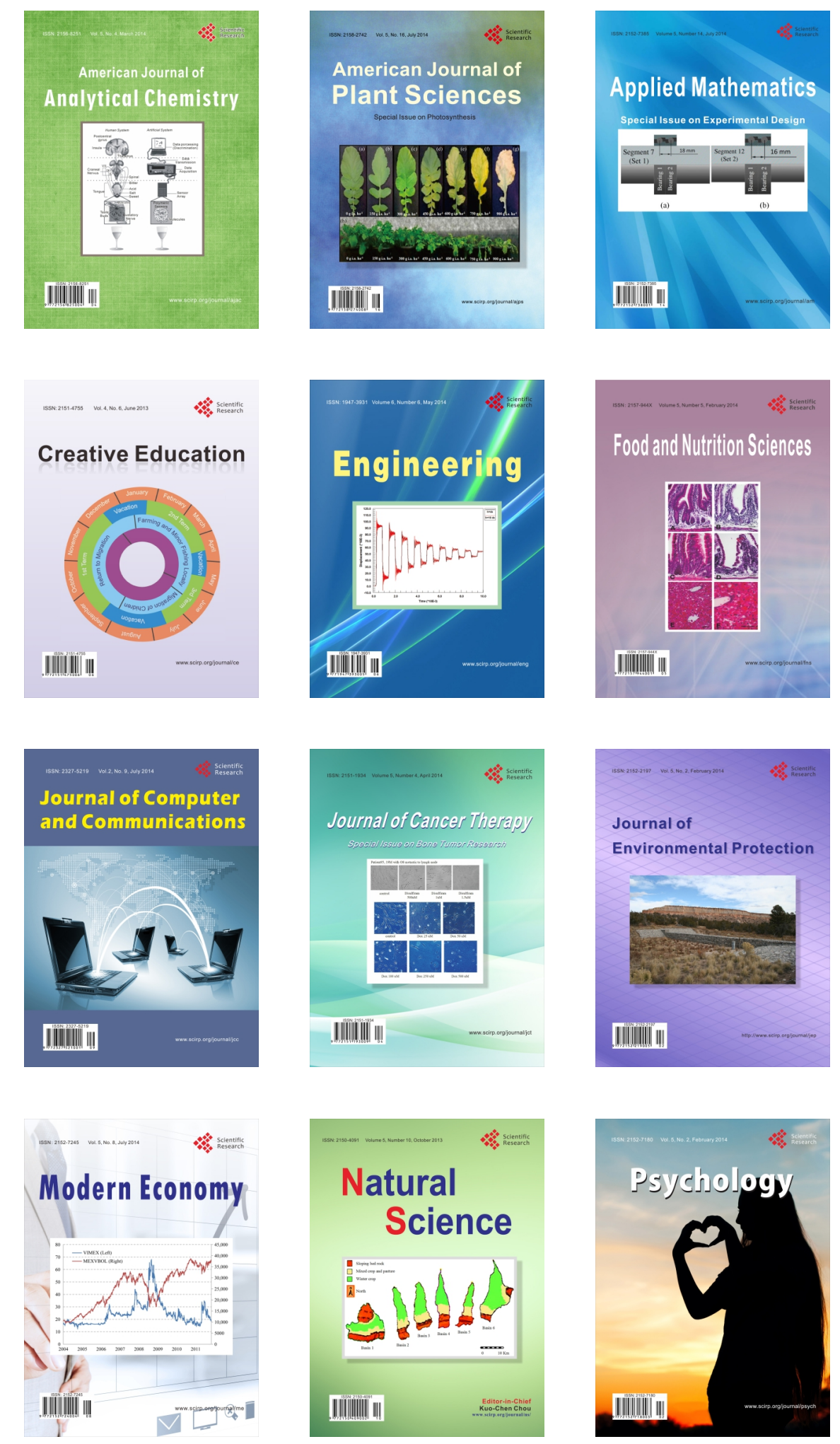